\title{
CONSUMO FOLIAR DE MILHO E DESENVOLVIMENTO DE LAGARTAS DE Spodoptera frugiperda (SMITH) PARASITADAS POR Chelonus insularis (CRESSON)(HYMENOPTERA: BRACONIDAE)
}

\author{
Maria A.A. Rezende' ${ }^{1}$ Ivan Cruz $^{2}$ e Terezinha M.C. Della Lucia ${ }^{1}$
}

\begin{abstract}
Foliar Consumption of Maize and Larval Development of Spodoptera frugiperda (Smith) Parasitized by Chelonus insularis (Cresson) (Hymenoptera: Braconidae)

Foliar consumption and larval development of non parasitized and parasitized Spodoptera frugiperda (Smith) larvae by Chelonus insularis (Cress.) were evaluated daily on maize leaves in the laboratory. Parasitized larvae consumed a total leaf area of $12.2 \mathrm{~cm}^{2}$ and feeding stopped on the $14^{\text {th }}$ observation day when they started pupation. Healthy larvae consumed a total of $179.7 \mathrm{~cm}^{2}$ and fed until the $18^{\text {th }}$ observation day. The total average length and the weight of parasitized larvae was $45.4 \%$ and $89.2 \%$, respectively, lower than healthy larvae.
\end{abstract}

KEY WORDS: Insecta, fall armyworm, damage, biological control, foliar consumption.

\section{RESUMO}

O consumo foliar e o desenvolvimento de lagartas de Spodoptera frugiperda (Smith) não parasitadas e parasitadas por Chelonus insularis (Cress.) foram avaliados diariamente, em folhas de milho, no laboratório. As lagartas parasitadas consumiram um total de $12,2 \mathrm{~cm}^{2} \mathrm{de}$ área foliar cessando sua alimentação no $14^{\circ}$ dia de observação quando iniciou-se o período de pré-pupa. Lagartas não parasitadas consumiram um total de $179,7 \mathrm{~cm}^{2}$ de área foliar, alimentando-se até o $18^{\circ}$ dia de observação. O comprimento total e o peso de lagartas parasitadas foram respectivamente, $45,4 \%$ e $89,2 \%$ menores que os mesmos parâmetros obtidos de lagartas sadias.

PALAVRAS-CHAVE: Insecta, lagarta do cartucho, danos, controle biológico, consumo foliar.

Recebido em 10/09/93. Aceito em 17/10/93.

${ }^{1}$ Universidade Federal de Viçosa, Departamento de Entomologia, 36570-000, Viçosa, MG.

${ }^{2}$ EMBRAPA/CNPMS, Caixa postal 151, 35700-000, Sete Lagoas, MG. 


\section{INTRODUÇÃO}

Têm sido observadas alterações causadas por microhimenópteros no crescimento e desenvolvimento do hospedeiro relacionados com as reduções em tamanho e peso, no número e duração dos ínstares, pupação e taxa de consumo alimentar (Lewis 1970, Rahman 1970, Vinson 1972 e Jones et al. 1981). Ables \& Vinson (1981) verificaram que lagartas de Heliothis virescens (Fabr.) e de Spodoptera ornithogalli (Guen.) parasitadas por Chelonus insularis (Cress.) ganharam menos peso que as não parasitadas. Este fato foi evidente no $5^{\circ}$ dia de parasitismo em $H$. virescens e no $7^{\circ}$ para $S$. ornithogalli. A larva do parasitóide emergiu de ambos hospedeiros no $13^{\circ}$ dia, apresentando taxas aparentemente similares de desenvolvimento. Grant \& Shetard (1984) observaram que a lagarta de Pseudoplusia includens (Walk.) quando parasitada pelo braconídeo Meteorus autographae (Mueseb.) cessou a alimentação do $6^{\circ}$ para $08^{\circ}$ dia depois de parasitada e consumiu $78 \%$ a menos de folha de sorgo do que as lagartas não parasitadas. Fato semelhante ocorreu com os parasitóides Campoletis sonorensis(Carls.) e Rogas laphygmae (Vier.) que reduziram a alimentação de suas lagartas hospedeiras (Isenhour 1988). A avaliação na alteração do padrão de crescimento na lagarta do cartucho Spodoptera frugiperda (Smith) depois de parasitadas por Apanteles marginiventris (Cress.), Campoletis grioti (Blanch.), C. insularis e Eiphosoma vitticole (Cress.) foi feita por Ashley (1983). A redução máxima no peso larval foi de 97; 96; 70 e $62 \%$, respectivamente.

Procurou-se determinar, neste trabalho, o consumo foliar e o desenvolvimento de lagartas $S$. frugiperda parasitadas por $C$. insularis a fim de avaliar a utilização deste parasitóide em um programa de manejo integrado da referida lagarta, na cultura do milho.

\section{MATERIAL E MÉTODOS}

O trabalho foi conduzido no Centro Nacional de Pesquisa de Milho e Sorgo/CNPMS da EMBRAPA, em Sete Lagoas, Minas Gerais e na Universidade Federal de Viçosa, Minas Gerais. A criação de $C$. insularis foi estabelecida a partir de adultos coletados em campo e mantidos em gaiolas de postura em laboratório, sob fotofase de $12 \mathrm{~h}$, a uma temperatura de 25 $\pm 2^{\circ} \mathrm{Ce} 73 \pm 2 \%$ de umidade relativa. As lagartas de $S$. frugiperda utilizadas como hospedeiro foram criadas em dieta artificial a base de feijão e germe de trigo (Perkins et al. 1973) sob as mesmas condições de laboratório.

Para as observações do consumo foliar, 18 lagartas recém-eclodidas parasitadas e 18 não parasitadas foram individualizadas em copos plásticos de $3,5 \times 5 \mathrm{~cm}$ de $50 \mathrm{ml}$ de capacidade e alimentadas com discos de folhas de milho. As medições a partir do $7^{\circ}$ dia de alimentação das lagartas quando começaram a aparecer sinais de alimentação nas folhas. Estas foram cortadas em discos de aproximadamente $12 \mathrm{~cm}^{2}$, os quais passavam em medidor de área foliar (Li-Cor) antes e depois de 24 horas após sua apresentação às lagartas. Simultaneamente eram medidas as testemunhas que consistiam em discos foliares preparados e medidos de maneira idêntica, porém sem as lagartas. Com estes, era obtida a quantidade da perda natural de água nas folhas para se calcular o consumo foliar real de $S$. frugiperda. A medida que as lagartas aumentavam o consumo foliar, acrescentou-se maior número de discos até o início da fase de pré-pupa dos hospedeiros. Os resultados foram comparados através do teste $t$ ao nível de $5 \%$ de significância. As fezes das lagartas foram acondicionadas individualmente em copinhos e secas em estufa a $35^{\circ} \mathrm{C}$ num período de $48 \mathrm{~h}$ para sua pesagem. Dezoito lagartas parasitadas e dezoito não parasitadas recém-eclodidas foram individualizadas e acondicionadas em copos plásticos, descritos anteriormente, contendo pedaços de folhas de milho que eram trocados 
diariamente para as observações do desenvolvimento das lagartas. Estes insetos foram mantidos no Insetário da Universidade Federal de Viçosa na temperatura de $25 \pm 2^{\circ} \mathrm{C}$, umidade relativa de $75 \pm 2 \%$ e fotoperíodo de $12 \mathrm{~h}$. A largura da cápsula cefálica e o comprimento de cada lagarta foram medidos sob lupa com ocular micrométrica e o peso individual obtido em balança de precisão diariamente até o início do período de pré-pupa do lepidóptero. Com esses dados foram determinados a diferença de crescimento e desenvolvimento das lagartas de $S$. frugiperda e o ínstar do hospedeiro em que ocorre a saída da larva de C. insularis.

Os dados foram comparados através do teste $t$ ao nível de $5 \%$ de significância, a partir do sétimo dia, já que o aparelho utilizado nas medições de área foliar não foi sensível para registrar o consumo das lagartas até o sexto dia, pois a maioria delas só havia raspado as folhas.

\section{RESULTADOS E DISCUSSÃO}

O consumo total das lagartas parasitadas até o sexto dia foi de $0,53 \mathrm{~cm}^{2}$ e das não parasitadas de $0,86 \mathrm{~cm}^{2}$. No $8^{\circ}$ e $10^{\circ}$ dias observou-se grande número de lagartas não parasitadas reduzindo a alimentação devido a troca de ínstar, o que não ocorreu a partir do $11^{\circ}$ ao $13^{\circ}$ dias (Fig. 1). O consumo foliar até o $13^{\circ}$ dia, que corresponde ao consumo total pelas lagartas parasitadas, foi de $12,2 \mathrm{~cm}^{2}$. Até esta data o consumo foliar para as lagartas não parasitadas foi de $60,9 \mathrm{~cm}^{2}$. O consumo total foi de $179,7 \mathrm{~cm}^{2}$, obtido no $17^{\circ}$ dia de alimentação. As lagartas parasitadas cessaram sua alimentação no $14^{\circ}$ dia quando iniciou-se o período de pré-pupa. O mesmo aconteceu no $18^{\circ}$ dia para as lagartas não parasitadas. Esses resultados assemelham-se aos obtidos por Grant \& Shepard (1984) para o parasitóide M. autographae e aos obtidos por Isenhour (1988) para $C$. sonorensis e $R$. laphygmae em $P$. includens e em $S$. frugiperda, respectivamente. A antecipação do período de pré-pupa, em que a lagarta parasitada por Chelonus inicia o processo de tecer um casulo precocemente já tinha sido verificada por Luginbill (1928) em $S$. frugiperda.

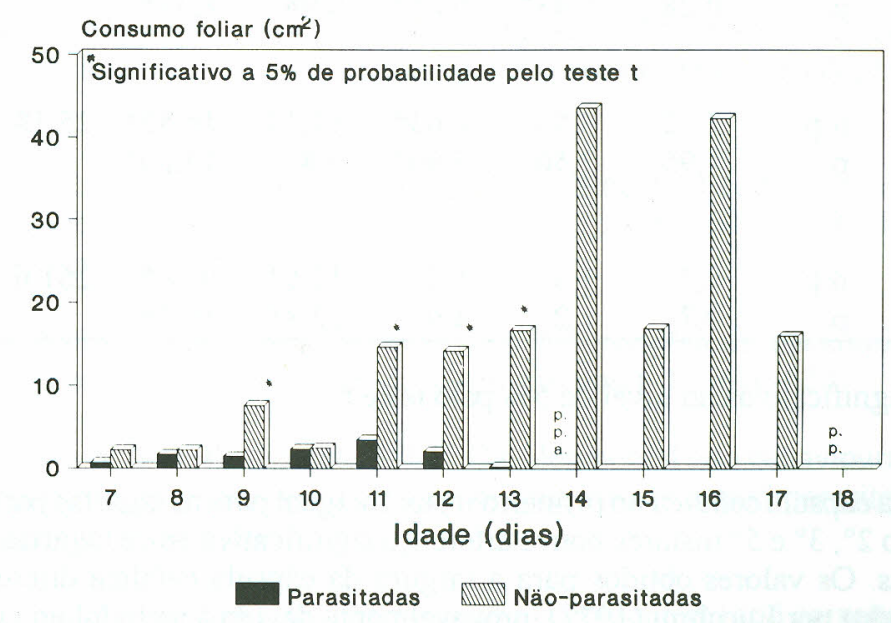

Figura 1. Consumo diário de folhas de milho $\left(\mathrm{cm}^{2}\right)$ por lagarta de Spodoptera frugiperda parasitadas ou não por Chelonus insularis (p.p. = início do período pré-pupa; p.p.a. = antecipação do período de pré-pupa). 
O peso total das fezes das lagartas parasitadas foi de $0,03 \mathrm{~g}$ e das não parasitadas de 0,38 g, tendo havido diferença significativa entre estes valores. Essa menor alimentação das lagartas significa, na prática, menor dano às plantas. Dados obtidos por Hopper \& King (1984), apontaram, também, menor peso de fezes de $S$. frugiperda parasitadas por $A$. marginiventris, C. grioti, C. insularis e E. vitticole.

Lagartas parasitadas desenvolveram-se até $05^{\circ}$ ínstar, enquanto das não parasitadas seis foram até $06^{\circ} \mathrm{e} 13$ até $07^{\circ}$ instar (Tabela 1). $O 1^{\circ}$ ínstar nas lagartas parasitadas foi o de maior duração, apresentando diferença significativa equivalente a 1,67 dias a mais do que nas não parasitadas que tiveram a maior duração no $6^{\circ}$ e $7^{\circ}$ ínstares. A duração total dos ínstares nas lagartas parasitadas foi de 12,81 dias e nas não parasitadas 16,21 dias. Houve pouca diferença em dias entre $\circ 2^{\circ}, 3^{\circ}$ e $4^{\circ}$ instares. $O 5^{\circ}$ ínstar no qual as lagartas parasitadas iniciaram $o$ período de pré-pupa foi mais longo, bem como $\circ 1^{\circ}, \circ 2^{\circ}$ e $04^{\circ}$ do que nas não parasitadas (Tabela 1).

Tabela 1. Parâmetros de desenvolvimento de lagartas de Spodoptera frugiperda parasitadas (p) e não parasitadas (n.p.) por Chelonus insularis.

\begin{tabular}{|c|c|c|c|c|c|c|c|c|}
\hline \multirow{2}{*}{ Parâmetros } & & \multicolumn{6}{|c|}{ Ínstares } & \multirow[b]{2}{*}{$7^{\circ}$} \\
\hline & & $1^{\circ}$ & $2^{\circ}$ & $3^{\circ}$ & $4^{\circ}$ & $5^{\circ}$ & $6^{\circ}$ & \\
\hline $\begin{array}{l}\text { Duração de } \\
\text { cada instar } \\
\text { (dias) }\end{array}$ & $\begin{array}{l}\text { n.p. } \\
\text { p. }\end{array}$ & $\begin{array}{l}2,53^{*} \\
4,20^{*}\end{array}$ & $\begin{array}{l}2,00 \\
2,15\end{array}$ & $\begin{array}{l}2,05 \\
1,89\end{array}$ & $\begin{array}{l}1,94 \\
2,21\end{array}$ & $\begin{array}{l}2,05 \\
2,36\end{array}$ & 2,79 & 2,85 \\
\hline $\begin{array}{l}\text { Cápsula } \\
\text { cefálica } \\
(\mathrm{mm})\end{array}$ & $\begin{array}{l}\text { n.p. } \\
\text { p. }\end{array}$ & $\begin{array}{l}0,28 \\
0,28\end{array}$ & $\begin{array}{l}0,48^{*} \\
0,43^{*}\end{array}$ & $\begin{array}{l}0,71^{*} \\
0,65^{*}\end{array}$ & $\begin{array}{l}1,11 \\
0,98\end{array}$ & $\begin{array}{l}1,62^{*} \\
1,33^{*}\end{array}$ & 2,32 & 2,66 \\
\hline $\begin{array}{l}\text { Comprimento } \\
\text { do corpo } \\
(\mathrm{mm})\end{array}$ & $\begin{array}{l}\text { n.p. } \\
\text { p. }\end{array}$ & $\begin{array}{l}2,12 \\
1,95\end{array}$ & $\begin{array}{l}3,53 \\
3,50\end{array}$ & $\begin{array}{l}6,63^{*} \\
5,80^{*}\end{array}$ & $\begin{array}{l}11,13 \\
9,80\end{array}$ & $\begin{array}{l}16,85^{*} \\
13,20^{*}\end{array}$ & 25,18 & 29,06 \\
\hline $\begin{array}{l}\text { Peso após } \\
3^{\circ} \mathrm{dia}(\mathrm{mg})\end{array}$ & $\begin{array}{l}\text { n.p. } \\
\text { p. }\end{array}$ & $\begin{array}{l}0,7 \\
0,7\end{array}$ & $\begin{array}{l}1,3 \\
1,2\end{array}$ & $\begin{array}{l}5,3 \\
4,9\end{array}$ & $\begin{array}{l}17,6^{*} \\
12,8^{*}\end{array}$ & $\begin{array}{l}90,4 * \\
30,3 *\end{array}$ & 261,6 & 310,0 \\
\hline
\end{tabular}

* Diferenças significativas ao nível de $5 \%$ pelo teste $\mathrm{t}$.

A largura da cápsula cefálica no primeiro ínstar foi igual para as lagartas parasitadas e não parasitadas. No $2^{\circ}, 3^{\circ}$ e $5^{\circ}$ ínstares houve diferença significativa entre lagartas parasitadas e não parasitadas. Os valores obtidos para a largura da cápsula cefálica diferem, em parte, daqueles relatados por Lucchini (1977), provavelmente devido à metodologia utilizada para realizar as medições da cápsula cefálica. As lagartas parasitadas tiveram comprimento do corpo semelhante ao das não parasitadas até o $2^{\circ}$ ínstar, e menor em todos os outros, exceto no $3^{\circ}$ e $5^{\circ}$ ínstares. O comprimento percentual médio total das lagartas parasitadas em relação ao das não parasitadas foi de apenas $45,4 \%$. 
No $1^{\circ}, 2^{\circ}$ e $3^{\circ}$ instares, as lagartas parasitadas tiveram peso semelhante ao das não parasitadas. Nos ínstares seguintes as diferenças foram evidenciadas. Até o seu último ínstar, pesaram $66,5 \%$ a menos do que as não parasitadas neste mesmo ínstar. Considerando o ciclo total, a redução do peso das lagartas parasitadas em relação às não parasitadas foi de $89,2 \%$. Ashley (1983) verificou uma redução de $70 \%$ do peso de $S$. frugiperda quando parasitadas por C. insularis.

Diferenças no peso larval, cápsula cefálica e comprimento entre lagartas parasitadas e não parasitadas foram também observadas por Jones et al. (1981) em Trichoplusia ni (Hubn.) cujo efeito se tornou evidente a partir do $4^{\circ}$ ínstar. Neste trabalho foi possível avaliar a influência de $C$. insularis no desenvolvimento e crescimento de $S$. frugiperda a partir do $5^{\circ}$ ínstar em que as lagartas apresentavam cutícula mais áspera e enrigecida. A antecipação do período de prépupa aqui observada ocorreu também em $T$. $n i$ quando parasitadas por Chelonus sp., tendo sido causada pela depressão anormal do nível do hormônio juvenil (Jones 1985). As implicações desse fenômeno são importantes, uma vez que efeitos similares de parasitismo em populações de campo auxiliariam a manter o nível de dano das lagartas abaixo do limiar econômico uma vez que lagartas parasitadas ficam menos tempo na planta e consomem menor área foliar.

\section{LITERATURA CITADA}

Ables, J.R. \& S.B. Vinson. 1981. Regulation of host larval development by the egg-larval endoparasitoid Chelonus insularis (Hymenoptera: Braconidae). Entomophaga 20: 453458.

Ashley, T.R. 1983. Growth pattern alterations in fall armyworm, Spodoptera frugiperda, larvae after parasitization by Apanteles marginiventris, Campoletis grioti, Chelonus insularis and Eiphosoma vitticole. Fla. Entomol. 66: 260-266.

Grant, J.F. \& M. Shepard. 1984. Laboratory biology of Meteorus autographae (Hymenoptera: Braconidae) an indigenous parasitoid of soybean looper(Lepidoptera: Noctuidae) larvae. Environ. Entomol. 13: 838-842.

Hopper, K.R. \& E.G. King. 1984. Feeding and movement on cotton of Heliothis species (Lepidoptera: Noctuidae) parasitized by Microplitis croceipes (Hymenoptera: Braconidae) Environ. Entomol. 13: 1654-1660.

Isenhour, D.J. 1988. Interactions between two hymenopterous parasitoids of the fall armyworm (Lepidoptera: Noctuidae). Environ. Entomol. 17: 616-620.

Jones, D., G. Jones \& B.D. Hammock. 1981. Developmental and behavioural responses of larvae Trichoplusia ni to parasitization by an imported braconid parasite Chelonus sp. Physiol. Entomol. 6: 387-394.

Jones, D. 1985. Endocrine interaction between host (Lepidoptera) and parasite (Cheloninae: Hymenoptera) is the host or the parasite in control? Ann. Entomol. Soc. Am. 78: 141-148.

Lewis, W.J. 1970. Study of species and instars of larval Heliothis parasitized by Microplitis croceipes. J. Econ. Entomol. 63: 363-365. 
Lucchini, F. 1977. Biologia de Spodoptera frugiperda (Lepidoptera: Noctuidae) (Smith, 1797). Níveis de prejuízos e avaliação toxicológica de inseticidas para o seu combate em milho. Tese de mestrado, Universidade Federal do Paraná, Curitiba, 114p.

Luginbill, P. 1928. The fall armyworm. Washington, USDA, Tech. Bull. 34, 91p.

Perkins, W.D., R.L. Sparks, D.R. Wiseman, J.W. Snow, W.W. McMillian. 1973. Artificial diet for mass rearing of corn earworm (Heliothis zea), ARS-USDA, Prod. Res. Rep. 154, 7p.

Rahman, M. 1970. Effect of parasitization on food consumption of Pieris rapae larvae. J. Econ. Entomol. 63: 820-821.

Vinson, S.B. 1972. Factors involved in successful attack Heliothis virescens on by the parasitoid Cardiochiles nigriceps. J. Inv. Pathol. 20: 118-123. 